DOI 10.1515/mjpaa-2017-0007

Volume 3(1), 2017, Pages 70-82

ISSN: 2351-8227

\title{
Infinitely Many Solutions of the Neumann Problem for Elliptic systems in Anisotropic Variable Exponent Sobolev Spaces
}

\section{A.AHMED ${ }^{1, a}$, M.S.B.ELEMINE VALL ${ }^{1, b}$ AND A.TOUZANI ${ }^{1, c}$}

ABSTRACT. In this paper, we prove the existence of infinitely many solutions for the following system

$$
\left\{\begin{array}{c}
\sum_{i=1}^{N} \frac{\partial}{\partial x_{i}} a_{i}\left(\cdot, \frac{\partial u}{\partial x_{i}}\right)+h(\cdot)|u|^{p_{0}(\cdot)-2} u=\theta(\cdot) f(u, v) \text { in } \Omega \\
\sum_{i=1}^{N} \frac{\partial}{\partial x_{i}} b_{i}\left(\cdot, \frac{\partial v}{\partial x_{i}}\right)+k(\cdot)|v|^{p_{0}(\cdot)-2} v=\theta(\cdot) g(u, v) \text { in } \Omega \\
\sum_{i=1}^{N} a_{i}\left(\cdot, \frac{\partial u}{\partial x_{i}}\right) \gamma_{i}=\sum_{i=1}^{N} b_{i}\left(\cdot, \frac{\partial v}{\partial x_{i}}\right) \gamma_{i}=0 \quad \text { on } \partial \Omega,
\end{array}\right.
$$

by applying a critical point variational principle obtained by Ricceri as a consequence of a more general variational principle and the theory of the anisotropic variable exponent Sobolev spaces. 2010 Mathematics Subject Classification. 35K05 - 35K55.

Key words and phrases. Neumann elliptic problem; Gradient system; weak solutions; Variational principle; Anisotropic variable exponent Sobolev spaces..

\section{Introduction}

Let $\Omega \subset \mathbb{R}^{N}$ be an open bounded set with boundary $\partial \Omega$ of class $C^{1}$, and let $\vec{\gamma}$ be the outward unit normal to $\partial \Omega$.

In these last years, the anisotropic variable exponent Sobolev space $W^{1, \vec{p}(\cdot)}(\Omega)$ have captured the attention of many researchers and a new operator has been introduced in the

Received January 15, 2017 - Accepted June 8, 2017.

(C)The Author(s) 2017. This article is published with open access by Sidi Mohamed Ben Abdallah University.

${ }^{1}$ University of Sidi Mohamed Ibn Abdellah, Faculty of Sciences Dhar El Mahraz, Laboratory LAMA, Department of Mathematics, B.P. 1796 Atlas Fez, Morocco

${ }^{a}$ e-mail: ahmedmath2001@gmail.com

${ }^{b}$ e-mail: saad2012bouh@gmail.com

c e-mail: atouzani07@gmail.com 
literature, namely

$$
\Delta_{\vec{p}(x)} u=\sum_{i=1}^{N} \frac{\partial}{\partial x_{i}}\left(\left|\frac{\partial u}{\partial x_{i}}\right|^{p_{i}(x)-2} \frac{\partial u}{\partial x_{i}}\right) .
$$

It's clear that this $\vec{p}(x)$-Laplace operator is a generalization of the $p(\cdot)$-Laplace operator. For some existing results of strongly nonlinear elliptic equations in the anisotropic variable exponent Sobolev spaces, see references [2, 10, 19].

In the present paper, we consider a problem involving a more general type of operator, that is,

$$
\sum_{i=1}^{N} \frac{\partial}{\partial x_{i}} a_{i}\left(x, \frac{\partial u}{\partial x_{i}}\right)
$$

the applications $a_{i}: \Omega \times \mathbb{R} \longrightarrow \mathbb{R}$ are Carathédory functions satisfying some hypothesis conditions for $\mathrm{i}=1, \ldots, \mathrm{N}$ (see Section 3 ). The operator defined above is of $\vec{p}(\cdot)$ )-Laplacian type when we take $a_{i}(x, s)=|s|^{p_{i}(x)-2} s$ for all $x \in \Omega, s \in \mathbb{R}$ and all $i=1, \ldots, N$.

Notice that the general operator given by (2) can admit degenerate and singular points. It is no surprising that there exist previous works treating problems with same operator, to give some examples, we refer the reader to $[8,12,13]$ where the authors were concerned with Dirichlet problems. Our work is focused on a Neumann problem, we refer the reader to [1,9].

In this paper, we are interested in the following problem

$$
(\mathcal{P})\left\{\begin{array}{c}
\sum_{i=1}^{N} \frac{\partial}{\partial x_{i}} a_{i}\left(\cdot, \frac{\partial u}{\partial x_{i}}\right)+h(\cdot)|u|^{p_{0}(\cdot)-2} u=\theta(\cdot) f(u, v) \quad \text { in } \Omega \\
\sum_{i=1}^{N} \frac{\partial}{\partial x_{i}} b_{i}\left(\cdot, \frac{\partial v}{\partial x_{i}}\right)+k(\cdot)|v|^{p_{0}(\cdot)-2} v=\theta(\cdot) g(u, v) \quad \text { in } \Omega \\
\sum_{i=1}^{N} a_{i}\left(\cdot, \frac{\partial u}{\partial x_{i}}\right) \gamma_{i}=\sum_{i=1}^{N} b_{i}\left(\cdot, \frac{\partial v}{\partial x_{i}}\right) \gamma_{i}=0 \quad \text { on } \partial \Omega .
\end{array}\right.
$$

More precisely we are interested to the existence of infinitely many weak solutions to such a problem.

In the context of Dirichlet boundary conditions J. Vlin have studied the existence of non trivial solutions of $(\mathcal{P})$ in the isotropic variable exponent Sobolev space, see [18]. In the same case M. Bendahmane and F. Mokhtari have studied in [5] the problem $(\mathcal{P})$ in the isotropic variable Sobolev space where the second term was a measure data. In the same framework, D. S. Moschetto in [15] have studied the problem $(\mathcal{P})$ in the particular case of homogeneous Neumann condition and $\sum_{i=1}^{N} \frac{\partial}{\partial x_{i}} a_{i}\left(\cdot, \frac{\partial u}{\partial x_{i}}\right)=\Delta_{\vec{p}(\cdot)}(u)$ and $\sum_{i=1}^{N} \frac{\partial}{\partial x_{i}} b_{i}\left(\cdot, \frac{\partial v}{\partial x_{i}}\right)=\Delta_{\vec{p}(\cdot)}(v)$.

Even though the problem $(\mathcal{P})$ has been studied by some other authors (see e.g. [3, 6, 7, $14,17,20])$ the hypotheses we use in this paper are totally different and so are our results.

The aim of the present paper is to generalize the results of $[1,5,15,18]$ in the anisotropic cases.

The main difficulties with this kind of problems are the framework of anisotropic Sobolev spaces and the fact that we have Neumann boundary conditions that make some difficulties in the application of theorem 1.1.

The following theorem plays an important role in this paper.

Theorem 1.1. (See [16], Theorem 2.5). Let $X$ be a reflexive real Banach space, and let $\Phi, \Psi: X \rightarrow \mathbb{R}$ be two sequentially weakly lower semicontinuous and Gâteaux differentiable functionals. Also, assume that $\Psi$ is (strongly) continuous and satisfies $\lim _{\|u\| \rightarrow+\infty} \Psi(u)=$ $+\infty$. For each $\rho>\inf _{X} \Psi$, put 


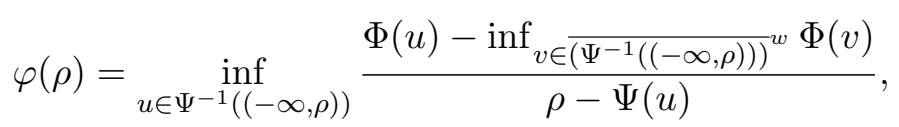

where ${\overline{\left(\Psi^{-1}((-\infty, \rho))\right)}}^{w}$ is the closure of $\Psi^{-1}((-\infty, \rho))$ in the weak topology. Furthermore, set

$$
\gamma=\liminf _{\rho \rightarrow+\infty} \varphi(\rho)
$$

and

$$
\delta=\liminf _{\rho \rightarrow\left(\inf _{X} \Psi\right)^{+}} \varphi(\rho) .
$$

then, the following conclusions hold:

(a) For each $\rho>\inf _{X} \Psi$ and each $t>\varphi(\rho)$, the functional $\Phi+t \Psi$ has a critical point which lies in $\Psi^{-1}((-\infty, \rho))$.

(b) If $\gamma<+\infty$, then, for each $t>\gamma$, the following alternative holds: either $\Phi+t \Psi$ has a global minimum, that is, it exists a sequence $\left\{u_{n}\right\}$ of critical points of $\Phi+t \Psi$ such that $\lim _{n \rightarrow \infty} \Psi\left(u_{n}\right)=+\infty$.

(c) If $\delta<+\infty$, then, for each $t>\delta$, the following alternative holds: either there exists a global minimum of $\Psi$ which is a local minimum of $\Phi+t \Psi$, or there exists a sequence of pairwise distinct critical points of $\Phi+t \Psi$ which weakly converges to a global minimum of $\Psi$.

This paper is organized as follows: In Section 2, we present some preliminary knowledge on the anisotropic Sobolev spaces with variable exponent. We introduce in Section 3 some assumptions for which our problem has solutions. In Section 4, we prove the existence of infinitely many weak solutions for our Neumann elliptic problem and we give a conclusion and some perspectives.

\section{Preliminaries}

Let $\Omega$ be an open bounded subset of $\mathbb{R}^{N}(N \geq 1)$, we define

$$
\mathcal{C}_{+}(\bar{\Omega})=\left\{\text { measurable function } z(\cdot): \bar{\Omega} \longmapsto \mathbb{R} \text { such that } 1<z^{-} \leq z^{+}<\infty\right\},
$$

where

$$
z^{-}=\operatorname{essinf}\{z(x) / x \in \Omega\} \quad \text { and } \quad z^{+}=\operatorname{ess} \sup \{z(x) / x \in \Omega\} .
$$

We define the Lebesgue space with variable exponent $L^{z(\cdot)}(\Omega)$ as the set of all measurable functions $u: \Omega \longmapsto \mathbb{R}$ for which the convex modular

$$
\rho_{z(\cdot)}(u):=\int_{\Omega}|u|^{z(x)} d x
$$

is finite, then

$$
\|u\|_{z(\cdot)}=\inf \left\{\lambda>0 \quad: \quad \rho_{z(\cdot)}(u / \lambda) \leq 1\right\}
$$

defines a norm in $L^{z(\cdot)}(\Omega)$, called the Luxemburg norm.

The space $\left(L^{z(\cdot)}(\Omega),\|\cdot\|_{z(\cdot)}\right)$ is a separable and reflexive Banach space. Moreover, the space $L^{z(\cdot)}(\Omega)$ is uniformly convex, hence reflexive, and its dual space is isomorphic to $L^{z^{\prime}(\cdot)}(\Omega)$, 
where $\frac{1}{z(x)}+\frac{1}{z^{\prime}(x)}=1$.

Finally, we have the Hölder type inequality:

$$
\left|\int_{\Omega} u v d x\right| \leq\left(\frac{1}{z^{-}}+\frac{1}{\left(z^{\prime}\right)^{-}}\right)\|u\|_{z(\cdot)}\|v\|_{z^{\prime}(\cdot)}
$$

for all $u \in L^{z(\cdot)}(\Omega)$ and $v \in L^{z^{\prime}(\cdot)}(\Omega)$.

An important role in manipulating the generalized Lebesgue spaces is played by the modular $\rho_{z(\cdot)}$ of the space $L^{z(\cdot)}(\Omega)$, we have the following result.

Proposition 2.1. (See [11]). If $u \in L^{z(\cdot)}(\Omega)$, then the following properties hold true:

(i): $\|u\|_{z(\cdot)}>1 \Rightarrow\|u\|_{z(\cdot)}^{z^{-}}<\rho_{z(\cdot)}(u)<\|u\|_{z(\cdot)}^{z^{+}}$

(ii): $\|u\|_{z(\cdot)}<1 \Rightarrow\|u\|_{z(\cdot)}^{p^{+}}<\rho_{z(\cdot)}(u)<\|u\|_{z(\cdot)}^{z^{-}}$.

The Sobolev spaces with variable exponent is defined by

$$
W^{1, z(\cdot)}(\Omega)=\left\{u \in L^{z(\cdot)}(\Omega) \quad \text { and } \quad|\nabla u| \in L^{z(\cdot)}(\Omega)\right\},
$$

equipped with the following norm

$$
\|u\|_{1, z(\cdot)}=\|u\|_{z(\cdot)}+\|\nabla u\|_{z(\cdot)}
$$

The space $\left(W^{1, z(\cdot)}(\Omega),\|\cdot\|_{1, z(\cdot)}\right)$ is a separable and reflexive Banach space.

Now, we present the anisotropic variable exponent Sobolev space used for the study of the main problem.

Let $z_{0}(\cdot), z_{1}(\cdot), \ldots, z_{N}(\cdot)$ be $N+1$ variable exponents in $\mathcal{C}_{+}(\bar{\Omega})$. We denote

$$
\vec{z}(\cdot)=\left\{z_{0}(\cdot), \ldots, z_{N}(\cdot)\right\}, D^{0} u=u \text { and } D^{i} u=\frac{\partial u}{\partial x_{i}} \quad \text { for } i=1, \ldots, N
$$

We define

$$
\underline{z}=\min \left\{z_{i}^{-}, \quad i=0,1, \ldots, N\right\} \quad \text { then } \quad \underline{z}>1
$$

and

$$
\bar{z}=\max \left\{z_{i}^{+}, \quad i=0,1, \ldots, N\right\} .
$$

The anisotropic Sobolev space with variable exponent $W^{1, \vec{z}(\cdot)}(\Omega)$ is defined as follows

$$
W^{1, \vec{z}(\cdot)}(\Omega)=\left\{u \in L^{z_{0}(\cdot)}(\Omega) \text { and } D^{i} u \in L^{z_{i}(\cdot)}(\Omega) \text { for } i=1,2, \ldots, N\right\},
$$

endowed with the norm

$$
\|u\|_{1, \vec{z}(\cdot)}=\sum_{i=0}^{N}\left\|D^{i} u\right\|_{z_{i}(\cdot)} .
$$

The space $\left(W^{1, \vec{z}(\cdot)}(\Omega),\|\cdot\|_{1, \vec{z}(\cdot)}\right)$ is separable and reflexive Banach space (cf. [4, 12]).

\section{Essential assumptions}

Let $p_{0}(\cdot), p_{1}(\cdot), \ldots, p_{N}(\cdot)$ and $q_{0}(\cdot), q_{1}(\cdot), \ldots, q_{N}(\cdot)$ be $2(N+1)$ variable exponents in $\mathcal{C}_{+}(\bar{\Omega})$. We assume that

$$
\underline{p}>N \text { and } \underline{q}>N
$$


Proposition 3.1. Since $W^{1, \vec{p}(\cdot)}(\Omega)$ (respectively $W^{1, \vec{q}(\cdot)}(\Omega)$ ) is continuously embedded in $W^{1, \underline{p}}(\Omega)$ (respectively $W^{1, \underline{q}}(\Omega)$ ), and since $W^{1, \underline{p}}(\Omega)$ and $W^{1, \underline{q}}(\Omega)$ are compactly embedded in $C^{0}(\bar{\Omega})$ (the space of continuous functions), thus the spaces $W^{1, \vec{p}(\cdot)}(\Omega)$ and $W^{1, \vec{q}(\cdot)}(\Omega)$ are compactly embedded in $C^{0}(\bar{\Omega})$.

Then we can set

$$
\begin{aligned}
C_{1} & =\sup _{u \in W^{1, \vec{p}(\cdot)}(\Omega) \backslash\{0\}} \frac{\|u\|_{\infty}}{\|u\|_{1, \vec{p}(\cdot)}} . \\
C_{2} & =\sup _{u \in W^{1, \vec{q}(\cdot)}(\Omega) \backslash\{0\}} \frac{\|u\|_{\infty}}{\|u\|_{1, \vec{q}(\cdot)}} .
\end{aligned}
$$

The applications $a_{i}, b_{i}: \Omega \times \mathbb{R} \longmapsto \mathbb{R}$ are Carathéodory functions which satisfy the following assumptions :

$\left(H_{1}\right)$ : The growth condition :

$$
\begin{array}{ll}
\left|a_{i}(x, s)\right| \leq \eta_{i}\left(c_{i}(x)+|s|^{p_{i}(x)-1}\right) & \text { for } \quad i=1, \ldots, N, \\
\left|b_{i}(x, s)\right| \leq \delta_{i}\left(d_{i}(x)+|s|^{q_{i}(x)-1}\right) & \text { for } \quad i=1, \ldots, N,
\end{array}
$$

where $c_{i}(\cdot)$ (respectively $d_{i}(\cdot)$ ) is a nonnegative function in $L^{p_{i}^{\prime}(\cdot)}(\Omega)$ (respectively in $\left.L^{q_{i}^{\prime}(\cdot)}(\Omega)\right), \eta_{i}$ and $\delta_{i}$ are positives constants.

$\left(H_{2}\right)$ : The coercivity condition : There exist four constants $\alpha_{1}, \beta_{1}, \alpha_{2}, \beta_{2}>0$ such that

$$
\begin{aligned}
& \alpha_{1}|s|^{p_{i}(x)} \leq a_{i}(x, s) s \leq \beta_{1} A_{i}(x, s), \\
& \alpha_{2}|s|^{q_{i}(x)} \leq b_{i}(x, s) s \leq \beta_{2} B_{i}(x, s),
\end{aligned}
$$

where the functions $A_{i}, B_{i}: \Omega \times \mathbb{R} \longmapsto \mathbb{R}$, are defined by

$$
A_{i}(x, s)=\int_{0}^{s} a_{i}(x, t) d t \quad \text { and } \quad B_{i}(x, s)=\int_{0}^{s} b_{i}(x, t) d t .
$$

$\left(H_{3}\right)$ : The monotonicity condition :

$$
\begin{aligned}
& \left(a_{i}(x, s)-a_{i}(x, t)\right)(s-t)>0, \text { for all } x \in \Omega, \forall s, t \in \mathbb{R} \text { with } t \neq s . \\
& \left(b_{i}(x, s)-b_{i}(x, t)\right)(s-t)>0, \text { for all } x \in \Omega, \forall s, t \in \mathbb{R} \text { with } t \neq s .
\end{aligned}
$$

$\left(H_{4}\right): h(\cdot), k(\cdot) \in L^{\infty}(\Omega)$ and $\theta(\cdot) \in \mathcal{C}(\bar{\Omega})$ such that there exist $h_{0}, k_{0}>0$ such that

$$
\text { ess } \inf _{x \in \Omega} h(x)>h_{0} \quad \text { and } \quad \text { ess } \inf _{x \in \Omega} k(x)>k_{0} .
$$

$\left(H_{5}\right): f, g \in \mathcal{C}\left(\mathbb{R}^{2}\right)$ such that the differential form $f(u, v) d u+g(u, v) d v$ be exact.

Remark 3.1. $\left(H_{5}\right)$ implies that exists $H: \mathbb{R}^{2} \longmapsto \mathbb{R}$ be the integral of the differential form $f(u, v) d u+g(u, v) d v$ such that $H(0,0)=0$.

Let $X$ be the Cartesian product between $W^{1, \vec{p}(\cdot)}(\Omega)$ and $W^{1, \vec{q}(\cdot)}(\Omega)$ Sobolev spaces with the norm $\|(u, v)\|_{X}=\sqrt{\|u\|_{1, \vec{p}(\cdot)}^{2}+\|v\|_{1, \vec{q}(\cdot)}^{2}}$ or another equivalent to it.

We introduce the functionals $\Psi(\cdot, \cdot), \Phi(\cdot, \cdot): W^{1, \vec{p}(\cdot)}(\Omega) \times W^{1, \vec{q}(\cdot)}(\Omega) \longmapsto \mathbb{R}$ by

$$
\Psi(u, v)=\sum_{i=1}^{N} \int_{\Omega} A_{i}\left(x, \frac{\partial u}{\partial x_{i}}\right) d x+\sum_{i=1}^{N} \int_{\Omega} B_{i}\left(x, \frac{\partial v}{\partial x_{i}}\right) d x
$$




$$
+\int_{\Omega} \frac{h(x)}{p_{0}(x)}|u|^{p_{0}(x)} d x+\int_{\Omega} \frac{k(x)}{q_{0}(x)}|v|^{q_{0}(x)} d x
$$

and

$$
\Phi(u, v)=-\int_{\Omega} F(x, u(x), v(x)) d x .
$$

where $F: \bar{\Omega} \times \mathbb{R} \times \mathbb{R} \longrightarrow \mathbb{R}$ be defined as $F(x, u, v)=\theta(x) H(u, v)$.

Lemma 3.1. ( see [9], [12]) The functionals $\Psi(\cdot, \cdot)$ and $\Phi(\cdot, \cdot)$ are well defined on $X$. In addition, $\Psi(\cdot, \cdot)$ and $\Phi(\cdot, \cdot)$ is of class $\mathcal{C}^{1}(X, \mathbb{R})$ and

$$
\begin{aligned}
\Psi^{\prime}(u, v)(w, \phi) & =\sum_{i=1}^{N} \int_{\Omega} a_{i}\left(x, \frac{\partial u}{\partial x_{i}}\right) \frac{\partial w}{\partial x_{i}} d x+\sum_{i=1}^{N} \int_{\Omega} b_{i}\left(x, \frac{\partial v}{\partial x_{i}}\right) \frac{\partial \phi}{\partial x_{i}} d x \\
& +\int_{\Omega} h(x)|u|^{p_{0}(x)-2} u w d x+\int_{\Omega} k(x)|v|^{q_{0}(x)-2} v \phi d x
\end{aligned}
$$

and

$$
\Phi^{\prime}(u, v)(w, \phi)=-\int_{\Omega} \theta(x)\left(\frac{\partial H}{\partial u}(u(x), v(x)) w(x)+\frac{\partial H}{\partial v}(u(x), v(x)) \phi(x)\right) d x .
$$

$\forall(u, v)(w, \phi) \in X$.

Lemma 3.2. (see [9]) Under the hypothesis $\left(H_{1}\right)-\left(H_{5}\right)$ and $(10)$ the functionals $\Psi(\cdot, \cdot)$ and $\Phi(\cdot, \cdot)$ are weakly lower semicontinuous.

Lemma 3.3. Under the hypothesis $\left(H_{1}\right)-\left(H_{5}\right)$ the functional $\Psi(\cdot, \cdot)$ is ceorcive, that is,

$$
\Psi(u, v) \longrightarrow+\infty \quad \text { as }\|(u, v)\|_{X} \longrightarrow+\infty \quad \text { for }(u, v) \in X .
$$

Proof Let $(u, v) \in X$, one has

$$
\begin{aligned}
\Psi(u, v) & =\sum_{i=1}^{N} \int_{\Omega} A_{i}\left(x, \frac{\partial u}{\partial x_{i}}\right) d x+\sum_{i=1}^{N} \int_{\Omega} B_{i}\left(x, \frac{\partial v}{\partial x_{i}}\right) d x \\
& +\int_{\Omega} \frac{h(x)}{p_{0}(x)}|u|^{p_{0}(x)} d x+\int_{\Omega} \frac{k(x)}{q_{0}(x)}|v|^{q_{0}(x)} d x
\end{aligned}
$$

then by using $\left(H_{2}\right)$ and $\left(H_{4}\right)$, we get

$$
\begin{aligned}
\Psi(u, v) & \geq \sum_{i=1}^{N} \int_{\Omega} \frac{\alpha_{1}}{\beta_{1}}\left|\frac{\partial u}{\partial x_{i}}\right|^{p_{i}(x)} d x+\int_{\Omega} \frac{h_{0}}{p_{0}^{+}}|u|^{p_{0}(x)} d x \\
& +\sum_{i=1}^{N} \int_{\Omega} \frac{\alpha_{2}}{\beta_{2}}\left|\frac{\partial v}{\partial x_{i}}\right|^{q_{i}(x)} d x+\int_{\Omega} \frac{k_{0}}{q_{0}^{+}}|v|^{q_{0}(x)} d x \\
& \geq \min \left(\frac{\alpha_{1}}{\beta_{1}}, \frac{h_{0}}{p_{0}^{+}}\right)\left[\sum_{i=1}^{N} \int_{\Omega}\left|\frac{\partial u}{\partial x_{i}}\right|^{p_{i}(x)} d x+\int_{\Omega}|u|^{p_{0}(x)} d x\right] \\
& +\min \left(\frac{\alpha_{2}}{\beta_{2}}, \frac{k_{0}}{q_{0}^{+}}\right)\left[\sum_{i=1}^{N} \int_{\Omega}\left|\frac{\partial v}{\partial x_{i}}\right|^{q_{i}(x)} d x+\int_{\Omega}|v|^{q_{0}(x)}\right] d x \\
& \left.\geq \min \left(\frac{\alpha_{1}}{\beta_{1}}, \frac{h_{0}}{p_{0}^{+}}\right)\left[\frac{1}{N^{\underline{p}-1}}\left(\sum_{i=1}^{N}\left\|\frac{\partial u}{\partial x_{i}}\right\|\right)_{p_{i}(\cdot)}\right)^{\underline{p}}+\|u\|_{p_{0}(\cdot)}^{\underline{p}}-N-1\right]
\end{aligned}
$$




$$
\begin{aligned}
& +\min \left(\frac{\alpha_{2}}{\beta_{2}}, \frac{k_{0}}{q_{0}^{+}}\right)\left[\frac{1}{N^{\underline{q}-1}}\left(\sum_{i=1}^{N}\left\|\frac{\partial v}{\partial x_{i}}\right\|_{q_{i}(\cdot)}\right)^{\underline{q}}+\|u\|_{q_{0}(\cdot)}^{\underline{q}}-N-1\right] \\
& \geq \min \left(\frac{\alpha_{1}}{\beta_{1}}, \frac{h_{0}}{p_{0}^{+}}\right)\left[\frac{1}{(2 N)^{\underline{p}-1}}\left(\sum_{i=1}^{N}\left\|\frac{\partial u}{\partial x_{i}}\right\|_{p_{i}(\cdot)}+\|u\|_{p_{0}(\cdot)}\right)^{\underline{p}}-N-1\right] \\
& +\min \left(\frac{\alpha_{2}}{\beta_{2}}, \frac{k_{0}}{q_{0}^{+}}\right)\left[\frac{1}{(2 N)^{\underline{q}-1}}\left(\sum_{i=1}^{N}\left\|\frac{\partial v}{\partial x_{i}}\right\|_{q_{i}(\cdot)}+\|u\|_{q_{0}(\cdot)}\right)^{\underline{q}}-N-1\right] \\
& =\frac{\min \left(\frac{\alpha_{1}}{\beta_{1}}, \frac{h_{0}}{p_{0}^{+}}\right)}{(2 N)^{\underline{p}-1}}\|u\|_{1, \vec{p}(\cdot)}+\frac{\min \left(\frac{\alpha_{2}}{\beta_{2}}, \frac{k_{0}}{q_{0}^{+}}\right)}{(2 N)^{\underline{q}-1}}\|u\|_{1, \vec{q}(\cdot)}-K_{2} \\
& \geq K_{1}\left(\|u\|_{1, \vec{q}(\cdot)}^{\underline{p}}+\|v\|_{1, \vec{q}(\cdot)}^{\underline{q}}\right)-K_{2} \\
& \geq K_{1}\|(u, v)\|_{X}-K_{2},
\end{aligned}
$$

where $K_{1}, K_{2}>0$ constants.

Thus, if $\|(u, v)\|_{X} \longrightarrow+\infty$ then $\Psi(u, v) \longrightarrow+\infty$.

Now, we set $\eta_{1}=\left(\frac{C_{1}}{h_{0} \text { meas }(\Omega)}\right)^{\bar{p}}$ and $\eta_{2}=\left(\frac{C_{2}}{k_{0} \text { meas }(\Omega)}\right)^{\bar{q}}$

$$
\alpha=\min \left\{\frac{1}{\bar{p} \eta_{1}^{\bar{p}}}, \frac{1}{\bar{p} \eta \frac{p}{1}}\right\}
$$

and

$$
\beta=\min \left\{\frac{1}{\bar{q} \eta_{2}^{\bar{q}}}, \frac{1}{\bar{q} \eta \frac{q}{2}}\right\}
$$

The sets $A(r), B(r), r>0$, below satisfied, play an important role in our exposition

$$
A(r)=\left\{(\xi, \eta) \in \mathbb{R}^{2} \text { such that } \alpha F_{\vec{p}(\cdot)}(\xi)+\beta F_{\vec{q}(\cdot)}(\eta) \leq r\right\}
$$

and

$$
B(r)=\left\{(\xi, \eta) \in \mathbb{R}^{2} \text { such that } \frac{1}{\underline{p}} \int_{\Omega} h(x) d x D_{\vec{p}(\cdot)}(\xi)+\frac{1}{\underline{q}} \int_{\Omega} k(x) d x D_{\vec{q}(\cdot)}(\eta) \leq r\right\}
$$

where $D_{\vec{r}(\cdot)}(t)=\max \left(|t|^{\bar{r}},|t|^{\underline{r}}\right)$ and $F_{\vec{r}(\cdot)}(t)=\min \left(|t|^{\bar{r}},|t|^{r}\right)$ with $\vec{r}(\cdot) \in\{\vec{p}(\cdot), \vec{q}(\cdot)\}$ and $t \in$ $\{\xi, \eta\}$.

Lemma 3.4. For all $r>0$, we have

$$
B(r) \subset A(r) .
$$

Proof We observe that, by the definition of constants $C_{1}$ and $C_{2}$, we have

$$
\|u\|_{\infty} \leq C_{1}\|u\|_{1, \vec{p}(\cdot)}, \forall u \in W^{1, \vec{p}(\cdot)}(\Omega)
$$

and

$$
\|v\|_{\infty} \leq C_{2}\|v\|_{1, \vec{q}(\cdot)}, \forall v \in W^{1, \vec{q}(\cdot)}(\Omega)
$$

For $u \equiv v \equiv 1$, we get

$$
1 \leq \eta_{1}^{\bar{p}} h_{0} \operatorname{meas}(\Omega) \leq \eta_{1}^{\bar{p}} \int_{\Omega} h(x) d x
$$


and

Thus, we obtain

$$
1 \leq \eta_{2}^{\bar{q}} k_{0} \operatorname{meas}(\Omega) \leq \int_{\Omega} \eta_{2}^{\bar{q}} k(x) d x
$$

$$
\alpha \leq \frac{1}{\bar{p} \eta_{1}^{\bar{p}}} \leq \frac{1}{\bar{p}} \int_{\Omega} h(x) d x, \quad \text { and } \beta \leq \frac{1}{\bar{q} \eta_{2}^{\bar{q}}} \leq \frac{1}{\bar{q}} \int_{\Omega} k(x) d x .
$$

Hence

Since

$$
\alpha \leq \frac{1}{\underline{p}} \int_{\Omega} h(x) d x, \quad \text { and } \beta \leq \frac{1}{\underline{q}} \int_{\Omega} k(x) d x .
$$

Thus, the inequality

$$
F_{\vec{p}(\cdot)}(t) \leq D_{\vec{p}(\cdot)}(t), \quad \text { and } F_{\vec{q}(\cdot)}(t) \leq D_{\vec{q}(\cdot)}(t), \forall t \in \mathbb{R} .
$$

$$
\alpha F_{\vec{p}(\cdot)}(\xi)+\beta F_{\vec{q}(\cdot)}(\eta) \leq \frac{1}{\underline{p}} \int_{\Omega} h(x) d x D_{\vec{p}(\cdot)}(\xi)+\frac{1}{\underline{q}} \int_{\Omega} k(x) d x D_{\vec{q}(\cdot)}(\eta) .
$$

holds for every $(\xi, \eta) \in \mathbb{R}^{2}$ and therefore the inclusion

$$
B(r) \subset A(r), \forall r>0
$$

holds.

\section{Main results}

Definition 4.1. We say that $(u, v) \in X$ a weak solution to the problem $(\mathcal{P})$ if for all $(w, \phi) \in$ $X$, we have

$$
\begin{aligned}
& \int_{\Omega}\left[\sum_{i=1}^{N} a_{i}\left(x, \frac{\partial u}{\partial x_{i}}(x)\right) \frac{\partial w}{\partial x_{i}}(x)+h(x)|u(x)|^{p_{0}(x)-2} u(x) w(x)\right] d x \\
& +\int_{\Omega}\left[\sum_{i=1}^{N} b_{i}\left(x, \frac{\partial v}{\partial x_{i}}(x)\right) \frac{\partial \phi}{\partial x_{i}}(x)+k(x)|v(x)|^{q_{0}(x)-2} v(x) \phi(x)\right] d x \\
& =\int_{\Omega} \theta(x)[f(u(x), v(x)) w(x)+g(u(x), v(x)) \phi(x)] d x,
\end{aligned}
$$

the weak solutions of $(\mathcal{P})$ are precisely critical points of $\Psi+\Phi$.

One of our main results is the following theorem

Theorem 4.1. Suppose that $\Psi(\cdot, \cdot)$ and $\Phi(\cdot, \cdot)$ are as in (13) and (14) and $\left(H_{1}\right)-\left(H_{5}\right)$ and (10) hold true.

(a): If there exist $\rho_{0}>0,\left(\xi_{0}, \eta_{0}\right) \in \mathbb{R}^{2}$ with $\left(\xi_{0}, \eta_{0}\right) \in \operatorname{Int}\left(B\left(\rho_{0}\right)\right)(\operatorname{Int}(B)$ is the interior of $B)$ and $\max _{A\left(\rho_{0}\right)} H(\xi, \eta)=H\left(\xi_{0}, \eta_{0}\right)$. Then, Problem $(\mathcal{P})$ admits a weak solution $(u, v) \in X$ such that $\Psi(u, v)<\rho_{0}$.

(b): If there exist a sequences, $\left(\rho_{n}\right)_{n} \subset \mathbb{R}^{+}$with $\rho_{n} \longrightarrow \infty$ as $n \longrightarrow+\infty$ and $\left(\xi_{n}\right)_{n},\left(\eta_{n}\right)_{n} \subset$ $\mathbb{R}$ such that $\left(\xi_{n}, \eta_{n}\right) \in \operatorname{Int}\left(B\left(\rho_{n}\right)\right)$ and $\max _{A\left(\rho_{n}\right)} H(\xi, \eta)=H\left(\xi_{n}, \eta_{n}\right), \forall n>0$ and if

$$
\limsup _{(\xi, \eta) \rightarrow+\infty} \frac{H(\xi, \eta) \int_{\Omega} \theta(x) d x}{D_{\vec{p}(\cdot)}(\xi) \int_{\Omega} h(x) d x+D_{\vec{q}(\cdot)}(\eta) \int_{\Omega} k(x) d x}>\max \left(\frac{1}{\underline{p}}, \frac{1}{\underline{q}}\right) .
$$


Then, the problem $(\mathcal{P})$ admits an unbounded sequence of a weak solutions in $X$.

(c): If there exist a sequences, $\left(\rho_{n}\right)_{n} \subset \mathbb{R}^{+}$with $\rho_{n} \longrightarrow 0$ as $n \longrightarrow+\infty$ and $\left(\xi_{n}\right)_{n},\left(\eta_{n}\right)_{n} \subset$

$\mathbb{R}$ such that $\left(\xi_{n}, \eta_{n}\right) \in \operatorname{Int}\left(B\left(\rho_{n}\right)\right)$ and $\max _{A\left(\rho_{n}\right)} H(\xi, \eta)=H\left(\xi_{n}, \eta_{n}\right), \forall n>0$ and if

$$
\limsup _{(\xi, \eta) \rightarrow(0,0)} \frac{H(\xi, \eta) \int_{\Omega} \theta(x) d x}{D_{\vec{p}(\cdot)}(\xi) \int_{\Omega} h(x) d x+D_{\vec{q}(\cdot)}(\eta) \int_{\Omega} k(x) d x}>\max \left(\frac{1}{\underline{p}}, \frac{1}{q}\right) .
$$

Then the problem $(\mathcal{P})$ admits a sequence of non zero weak solutions which strongly converges to $(u, v)$ in $X$.

\section{Proof}

Step 1: Proof of assertion (a). We apply the part $a$ of theorem 1.1 for showing that $\varphi\left(\rho_{0}\right)=0$ ( here $\varphi$ is the function defined in the theorem 1.1 and $t=1$ is assumed).

First, we observe that $\forall(u, v) \in \Psi^{-1}(]-\infty, \rho_{0}[)$, one has

$$
\begin{aligned}
0 \leq \varphi\left(\rho_{0}\right)= & \inf _{\Psi^{-1}(]-\infty, \rho_{0}[)} \frac{\Phi(u, v)-\frac{\inf }{\left(\Psi^{-1}(]-\infty, \rho_{0}[)\right)} w}{\rho_{0}-\Psi(u, v)} \Phi(u, v) \\
& \leq \frac{\inf _{(u, v)-} \Phi(u, v)}{\rho^{-1(]-\infty, \rho_{0}[)} w}{ }^{\rho_{0}-\Psi(u, v)}
\end{aligned}
$$

Let $u_{0}(x)=\xi_{0}, v_{0}(x)=\eta_{0}, \forall x \in \Omega$, then $\nabla u_{n}=\nabla v_{0}=0$ and Since $\left(\xi_{0}, \eta_{0}\right) \in \operatorname{Int}\left(B\left(\rho_{0}\right)\right)$, one has

$$
\begin{aligned}
\Psi\left(u_{0}, v_{0}\right) & =\int_{\Omega}\left[\frac{1}{p_{0}(x)} h(x)\left|\xi_{0}\right|^{p_{0}(x)}+\frac{1}{q_{0}(x)} k(x)\left|\eta_{0}\right|^{q_{0}(x)}\right] d x \\
& \leq \frac{1}{\underline{p}} \int_{\Omega} h(x) d x D_{\vec{p}(\cdot)}\left(\xi_{0}\right)+\frac{1}{\underline{q}} \int_{\Omega} k(x) d x D_{\vec{q}(\cdot)}\left(\eta_{0}\right)<\rho_{0} .
\end{aligned}
$$

Then, for almost every $x \in \bar{\Omega}$ and $\forall(u, v) \in{\overline{\Psi^{-1}(]-\infty, \rho_{0}[)}}^{w}$, one has

$$
\alpha F_{\vec{p}(\cdot)}(u(x))+\beta F_{\vec{q}(\cdot)}(v(x)) \leq \Psi(u, v) \leq \rho_{0} .
$$

The first inequality in (19) is obtained by the proposition 2.1, while the second inequality in (19) follows from the fact that $\left.\left.\overline{\Psi^{-1}(]-\infty, \rho_{0}[)}=\Psi^{-1}(]-\infty, \rho_{0}\right]\right)$.

Thu, since $(u(x), v(x)) \in A\left(\rho_{0}\right)$ and $H(u(x), v(x)) \leq H\left(\xi_{0}, \eta_{0}\right), \forall x \in \bar{\Omega}$.

Hence $-\Phi(u, v) \leq-\Phi\left(u_{0}, v_{0}\right) \forall(u, v) \in \overline{\Psi^{-1}(]-\infty, \rho_{0}[)}$. because,

$$
-\Phi\left(u_{0}, v_{0}\right)=\sup _{\Psi^{-1}(]-\infty, \rho_{0}[)}(-\Phi(u, v))=-\inf _{\Psi^{-1}(]-\infty, \rho_{0}[)_{w}} \Phi(u, v),
$$

and since $\Phi\left(u_{0}, v_{0}\right)<\rho_{0}$, it follows that

$$
\Phi\left(u_{0}, v_{0}\right)-\inf _{\Psi^{-1}(]-\infty, \rho_{0}[)} w(u, v)=\Phi\left(u_{0}, v_{0}\right)-\Phi\left(u_{0}, v_{0}\right)=0 .
$$

Then, by choosing $(u, v)=\left(u_{0}, v_{0}\right)$ in the inequality (18), one has $\varphi\left(\rho_{0}\right)=0$.

The conclusion $(a)$ of the theorem 1.1 assures that there is a critical point of $\Psi+\Phi$. 
Step 2: Proof of assertion (b). We apply the part (b) of theorem 1.1.

From the part $(a)$. we know that $\varphi\left(\rho_{n}\right)=0, \forall n \in \mathbb{N}$.

Then, since $\lim _{n \rightarrow \infty} \rho_{n}=+\infty$, one has

$$
\liminf _{\rho \rightarrow \infty} \varphi(\rho) \leq \liminf _{n \rightarrow \infty} \varphi\left(\rho_{n}\right)=0<1=t .
$$

Now, we fix $h$ satisfying that

$$
\limsup _{(\xi, \eta) \rightarrow+\infty} \frac{H(\xi, \eta) \int_{\Omega} \theta(x) d x}{D_{\vec{p}(\cdot)}(\xi) \int_{\Omega} h(x) d x+D_{\vec{q}(\cdot)}(\eta) \int_{\Omega} k(x) d x}>h>\max \left(\frac{1}{\underline{p}}, \frac{1}{q}\right),
$$

and we choose a sequence $\left(r_{n}, t_{n}\right)_{n}$ in $\mathbb{R}^{2}$ such that $\sqrt{r_{n}^{2}+t_{n}^{2}} \geq n$ and

$$
H\left(r_{n}, t_{n}\right) \int_{\Omega} \theta(x) d x>h\left(D_{\vec{p}(\cdot)}\left(r_{n}\right) \int_{\Omega} h(x) d x+D_{\vec{q}(\cdot)}\left(t_{n}\right) \int_{\Omega} k(x) d x\right), \quad \forall n \in \mathbb{N} .
$$

If we denote by $u_{n}$ and $v_{n}$ the constant functions on $\Omega$ which take the $r_{n}$ and $t_{n}$ values respectively, we have

$$
\begin{aligned}
\Phi\left(u_{n}, v_{n}\right)+\Psi\left(u_{n}, v_{n}\right) & =\Phi\left(r_{n}, t_{n}\right)+\Psi\left(r_{n}, t_{n}\right) \\
& =-\int_{\Omega} F\left(x, r_{n}, t_{n}\right) d x+\int_{\Omega} \frac{1}{p_{0}(x)} h(x)\left|r_{n}\right|^{p_{0}(x)} d x \\
& +\int_{\Omega} \frac{1}{q_{0}(x)} k(x)\left|t_{n}\right|^{q_{0}(x)} d x \\
& \leq \int_{\Omega}\left(\frac{1}{p}-h\right) h(x) D_{\vec{p}(\cdot)}\left(r_{n}\right) d x \\
& +\int_{\Omega}\left(\frac{1}{q}-h\right) h(x) D_{\vec{q}(\cdot)}\left(t_{n}\right) d x<0, \quad \forall n \in \mathbb{N} .
\end{aligned}
$$

Since $\left(\sqrt{r_{n}^{2}+t_{n}^{2}}\right)_{n}$ is unbounded, at least one of the two sequences $\left(r_{n}\right)_{n}$ or $\left(t_{n}\right)_{n}$ admits one divergent subsequence.

Hence $\left(D_{\vec{p}(\cdot)}\left(r_{n}\right)\right)_{n}$ and $\left(D_{\vec{q}(\cdot)}\left(t_{n}\right)\right)_{n}$ admit one divergent subsequence, thus, the functional $\Phi+\Psi$ is unbounded from below.

The conclusion $(b)$ of the theorem 1.1 assures that there is a sequence $\left(x_{n}, y_{n}\right)_{n}$ of critical points of $\Phi+\Psi$ such that $\lim _{n \rightarrow+\infty} \Psi\left(x_{n}, y_{n}\right)=+\infty$.

Moreover, since $\Psi$ is bounded on each bounded subset of $X$, the sequence $\left(x_{n}, y_{n}\right)_{n}$ must be unbounded in $X$.

Step 3: Proof of assertion (c). We apply the part (c) of theorem 1.1. As before, from the $(a)$. we know that $\varphi\left(\rho_{n}\right)=0, \forall n \in \mathbb{N}$.

Therefore after observing that $\inf _{X} \Psi=\Psi(u, v)=0$, since $\lim _{n \rightarrow \infty} \rho_{n}=0$, we have

$$
\delta=\liminf _{\rho \rightarrow 0^{+}} \varphi(\rho) \leq \liminf _{n \rightarrow+\infty} \varphi\left(\rho_{n}\right)=0<1=t .
$$

Now, we fix $h$ satisfying

$$
\limsup _{(\xi, \eta) \rightarrow(0,0)} \frac{H(\xi, \eta) \int_{\Omega} \theta(x) d x}{D_{\vec{p}(\cdot)}(\xi) \int_{\Omega} h(x) d x+D_{\vec{q}(\cdot)}(\eta) \int_{\Omega} k(x) d x}>h>\max \left(\frac{1}{\underline{p}}, \frac{1}{\underline{q}}\right),
$$

and choose a sequence $\left(\left(r_{n}, t_{n}\right)\right)_{n}$ in $\mathbb{R}^{2} \backslash\{(0,0)\}$ such that $\sqrt{r_{n}^{2}+t_{n}^{2}} \leq \frac{1}{n}$ and

$$
H\left(r_{n}, t_{n}\right) \int_{\Omega} \theta(x) d x>h\left(\left|r_{n}\right|^{\underline{p}} \int_{\Omega} h(x) d x+\left|t_{n}\right|^{\underline{q}} \int_{\Omega} k(x) d x\right), \quad \forall n \in \mathbb{R} .
$$


Once more if we denote by $u_{n}$ and $v_{n}$ the constant functions on $\Omega$ which equal $r_{n}$ and $t_{n}$ respectively.

Then, from proposition 2.1 the sequence $\left(\left(u_{n}, v_{n}\right)\right)_{n}$ strongly converges to $(u, v)$ in $X$ and one has

$$
\begin{aligned}
\Phi\left(u_{n}, v_{n}\right)+\Phi\left(u_{n}, v_{n}\right) & =\Phi\left(r_{n}, t_{n}\right)+\Psi\left(r_{n}, t_{n}\right) \\
& \leq \int_{\Omega}\left|r_{n}\right| \underline{\underline{p}}\left(\frac{1}{\underline{p}}-h\right) h(x) d x \\
& +\left|r_{n}\right| \underline{q} \int_{\Omega}\left(\frac{1}{q}-h\right) h(x) d x<0 \quad \forall n \in \mathbb{N} .
\end{aligned}
$$

Since $\Phi(u, v)+\Psi(u, v)=0$ in virtue of the last inequality $(u, v)$ can't be a local minimum of $\Phi+\Psi$.

Then, since $(u, v)$ is the only global minimum of $\Psi$, the conclusion $(c)$ of the theorem 1.1 assures that there is a sequence of pairwise distinct critical points of $\Phi+\Psi$ such that $\lim _{n \rightarrow \infty} \Psi\left(x_{n}, y_{n}\right)=0$ with $x_{n}, y_{n} \rightarrow 0$, thus $\left(x_{n}, y_{n}\right)_{n}$ must be in norm infinitesimal.

The following theorem is a practicable form of theorem 4.1 part (b).

Theorem 4.2. Let $\left(a_{n}\right)_{n}$ and $\left(b_{n}\right)_{n}$ be two sequences in $\mathbb{R}^{+}$satisfying

$$
b_{n}<a_{n} \quad \forall n \in \mathbb{N}, \lim _{n \rightarrow+\infty} b_{n}=+\infty, \quad \lim _{n \rightarrow+\infty} \frac{a_{n}}{b_{n}}=+\infty
$$

and let

$$
\begin{aligned}
A_{n} & =\left\{(\xi, \eta) \in \mathbb{R}^{2}: F_{\vec{p}(\cdot)}(\xi)+F_{\vec{q}(\cdot)}(\eta) \leq a_{n}\right\} \\
B_{n} & =\left\{(\xi, \eta) \in \mathbb{R}^{2}: D_{\vec{p}(\cdot)}(\xi)+D_{\vec{q}(\cdot)}(\eta) \leq b_{n}\right\},
\end{aligned}
$$

be such that $\sup _{A_{n} \backslash \text { Int } B_{n}} H \leq 0$ for all $n \in \mathbb{N}$.

Finally, let us assume that

$$
\limsup _{(\xi, \eta) \rightarrow+\infty} \frac{H(\xi, \eta) \int_{\Omega} \theta(x) d x}{D_{\vec{p}(\cdot)}(\xi) \int_{\Omega} h(x) d x+D_{\vec{q}(\cdot)}(\eta) \int_{\Omega} k(x) d x}>\max \left(\frac{1}{\underline{p}}, \frac{1}{\underline{q}}\right) .
$$

Then, Problem $(\mathcal{P})$ admits an unbounded sequence of weak solutions in $X$.

Proof Since $b_{n}<a_{n}$ it follows that $B_{n} \subseteq A_{n}$.

Let

$$
\gamma^{\prime}=\min \{\alpha, \beta\}>0 \quad \text { and } \quad \delta^{\prime}=\max \left\{\frac{\int_{\Omega} h(x) d x}{\underline{p}}, \frac{\int_{\Omega} k(x) d x}{\underline{q}}\right\}>0 .
$$

Then $\frac{\delta^{\prime}}{\gamma^{\prime}}>0$ and in virtue of $\lim _{n \rightarrow+\infty} \frac{a_{n}}{b_{n}}=+\infty$, then we get $\frac{\delta^{\prime}}{\gamma^{\prime}}<\frac{a_{n}}{b_{n}}$ for $n \in \mathbb{N}$ large enough.

Let $\rho_{n}=\gamma^{\prime} a_{n}$. Then $\left\{\rho_{n}\right\}_{n} \subset \mathbb{R}^{+}$is a divergent sequence and for $n$ large enough, the following inclusions hold

$$
\text { Int } B_{n} \subseteq B_{n} \subseteq B\left(\rho_{n}\right) \subseteq A\left(\rho_{n}\right) \subseteq A_{n},
$$

Then, since $H$ is negative in the set $A_{n} \backslash \operatorname{Int} B_{n}$ for all $n \in \mathbb{N}$, we have

$$
\max _{\text {IntB } B_{n}} H=\max _{A_{n}} H
$$


in particular, we have $\max _{\operatorname{Int} B_{n}} H=\max _{A\left(\rho_{n}\right)} H$ for $n \in \mathbb{N}$ large enough, i.e. there exist at least one sequence $\left(\xi_{n}, \eta_{n}\right)_{n} \subset \operatorname{Int} B_{n}$ such that for $n$ large enough, we have

$$
\max _{A\left(\rho_{n}\right)} H(\xi, \eta)=H\left(\xi_{n}, \eta_{n}\right)
$$

Thus, the sequences $\left(\xi_{n}\right)_{n},\left(\eta_{n}\right)_{n}$ and $\left(\rho_{n}\right)_{n}$ have got the properties required in theorem 4.1 part (b).

This completes the proof.

The following theorem is a practicable form of theorem 4.1 part (c).

Theorem 4.3. Let $\left(a_{n}\right)_{n}$ and $\left(b_{n}\right)_{n}$ be two sequences in $\mathbb{R}^{+}$satisfying

$$
b_{n}<a_{n} \quad \forall n \in \mathbb{N}, \lim _{n \rightarrow+\infty} a_{n}=0, \quad \lim _{n \rightarrow+\infty} \frac{a_{n}}{b_{n}}=+\infty
$$

and let

$$
\begin{array}{r}
A_{n}=\left\{(\xi, \eta) \in \mathbb{R}^{2}: F_{\vec{p}(\cdot)}(\xi)+F_{\vec{q}(\cdot)}(\eta) \leq a_{n}\right\} \\
B_{n}=\left\{(\xi, \eta) \in \mathbb{R}^{2}: D_{\vec{p}(\cdot)}(\xi)+D_{\vec{q}(\cdot)}(\eta) \leq b_{n}\right\},
\end{array}
$$

be such that $\sup _{A_{n} \backslash \text { IntB }} H \leq 0$ for all $n \in \mathbb{N}$.

Finally, let us assume that

$$
\limsup _{(\xi, \eta) \rightarrow(0,0)} \frac{H(\xi, \eta) \int_{\Omega} \theta(x) d x}{D_{\vec{p}(\cdot)}(\xi) \int_{\Omega} h(x) d x+D_{\vec{q}(\cdot)}(\eta) \int_{\Omega} k(x) d x}>\max \left(\frac{1}{\underline{p}}, \frac{1}{q}\right) .
$$

Then, Problem $(\mathcal{P})$ admits a sequence of non-zero weak solutions which strongly converges to $(u, v)$ in $X$.

Proof Likewise, by applying theorem 4.1 part (c), we get the theorem 4.3, whose proof will be omitted.

\section{Conclusion and perspective}

In this paper we have proved an existence result of infinitely many solutions of the elliptic problem $(\mathcal{P})$ in the anisotropic variable exponent Sobolev spaces by applying a variational principe of Ricceri, our future works will be devoted to the existence of infinitely many solutions elliptic and parabolic problems in the case of flux boundary conditions and the framework of anisotropic Sobolev spaces.

\section{References}

[1] A. Ahmed, T.Ahmedatt, H. Hjiaj and A. Touzani, Existence of infinitly many weak solutions for some quasilinear $\vec{p}(\cdot)$-elliptic Neumann problems, Mathematica Bohemica DOI: 10.21136/MB.2017.0037-15.

[2] A. Ahmed, H. Hjiaj and A. Touzani, Existence of infinitely many weak solutions for a Neumann elliptic equations involving the $\vec{p}(x)$-laplacian operator, Rend. Circ. Mat. Palermo DOI 10.1007/s12215-015-0210-1.

[3] A. Bechah, Local and global estimates for solutions of systems involving the p-Laplacian in unbounded domains, Electronic Journal of differential equations, 19 (2001), pp. 1-14. 
[4] M. Bendahmane, M. Chrif and S. El Manouni, An Approximation Result in Generalized Anisotropic Sobolev Spaces and Application, Z. Anal. Anwend., 30 (2011), no. 3, 341-353.

[5] M. Bendahmane, F. Mokhtari, Nonlinear elliptic systems with variable exponents and measure data, Moroccan J. Pure and Appl. Anal.(MJPAA) Volume 1(2), 2015, Pages 108-125.

[6] A. Bensedik, M. Bouchekif, On certain nonlinear elliptic systems with inde finite terms, Electronic Journal of differential equations, 83 (2002), pp. 1-16.

[7] L. Boccardo, D. Guedes De Figueiredo, Some Remarks on a system of quasilinear elliptic equations, Nonlinear differ. equ. appl., 9 (2002), pp. 309-323.

[8] M. M. Boureanu, Infinitely many solutions for a class of degenerate anisotropic elliptic problems with variable exponent, Taiwanese J. Math. 15 (2011) 2291-2310.

[9] M. M. Boureanu, V.D. Radulescu, Anisotropic Neumann problems in Sobolev spaces with variable exponent, Nonlinear Analysis (2011), doi:10.1016/j.na.2011.09.033.

[10] M. M. Boureanu, C. Udrea and D. N. Udrea, Anisotropic problems with variable exponents and constant Dirichlet condition, Electron. J. Diff. Equ., 2013 (2013), 1-13.

[11] X. L. Fan, D. Zhao, On the generalised Orlicz-Sobolev Space $W^{k, p(x)}(\Omega)$, J. Gansu Educ. College12(1)(1998) 1-6.

[12] B. Kone, S. Ouaro, S. Traore, Weak solutions for anisotropic nonlinear elliptic equations with variable exponents, Electron. J. Differential Equations 2009 (144) (2009) 1-11.

[13] M. Mihăilescu, G. Morosanu, Existence and multiplicity of solutions for an anisotropic elliptic problem involving variable exponent growth conditions, Appl. Anal. 89 (2010) 257271.

[14] D. S. Moschetto, Infintly many solutions to the Dirichlet problem for quasilinear elliptic systems involving the $p(x)$ and $q(x)$ Laplacien, Le matematiche Vol . LXIII (2008)Fasc. I, pp. 223-233.

[15] D. S. Moschetto, Infinitely Many Solutions to the Neumann Problem for Quasilinear Elliptic Systems Involving the $p(x)$ and $q(x)$-Laplacian, International Mathematical Forum, 4, 2009, no. 24, 1201-1211.

[16] B. Ricceri, A general variational principle and some of its applications, J. Comput. Appl.Math. 113 (2000) 401-410.

[17] D. Terman, Radial solutions of an elliptic system: solutions with a prescribed winding number, Houston Journal of mathematics, 15 (3) (1989).

[18] J. Vélin, Existence result for a gradient-type elliptic system involving a pair of $p(x)$ and $q(x)$-Laplacian operators, Complex Variables and Elliptic Equations, 2015, http://dx.doi.org/10.1080/17476933.2015.1110578

[19] L. Zhao, P. Zhao, X. Xie, Existence and multiplicity of solutions for divergence type elliptic equations, Electronic Journal of Differential Equations 2011 (2011) no. 43, 1-9.

[20] W. Zou,S. Li, J. Q. Liu, Nontrivial solutions for resonant cooperative elliptic systems via computations of critical groups, Nonlinear Analysis, 38 (1999) pp. 229-247. 